\title{
Characterization and Electromagnetic Studies on NiZn and NiCuZn Ferrites Prepared by Microwave Sintering Technique
}

\author{
Matli Penchal Reddy ${ }^{1}$, Il Gon Kim ${ }^{1 *}$, Dong Sun Yoo ${ }^{1}$, Wuppati Madhuri ${ }^{2}$, \\ Nagireddy Ramamanohar Reddy ${ }^{3}$, Kota Venkata Siva Kumar ${ }^{2}$, Rajuru Ramakrishna Reddy ${ }^{2}$ \\ ${ }^{1}$ Department of Physics, Changwon National University, Changwon, South Korea; ${ }^{2}$ Department of Physics, Sri Krishnadevaraya \\ University, Anantapur, India; ${ }^{3}$ Department of Materials Science and Nanotechnology, Yogi Vemana University, Kadapa, India. \\ Email: ${ }^{*}$ igkim@changwon.ac.kr, ${ }^{*}$ drlpenchal@gmail.com
}

Received April 24 $4^{\text {th }}, 2012$; revised June $3^{\text {rd }}, 2012$; accepted July $14^{\text {th }}, 2012$

\begin{abstract}
The low-temperature sintered NiZn and NiCuZn ferrites with the composition of $\mathrm{Ni}_{0.40} \mathrm{Zn}_{0.60} \mathrm{Fe}_{2} \mathrm{O}_{4}$ and $\mathrm{Ni}_{0.35} \mathrm{Cu}_{0.05} \mathrm{Zn}_{0.60}$ $\mathrm{Fe}_{2} \mathrm{O}_{4}$ were respectively synthesized by the microwave sintering method. These powders were calcined, compacted and sintered at $950^{\circ} \mathrm{C}$ for $30 \mathrm{~min}$. X-ray diffraction (XRD) patterns of the samples indicate the formation of single-phase cubic spinel structure. The grain size was estimated from SEM images which increase with $\mathrm{CuO}$ addition. The X-ray density is higher than the bulk density in both the ferrites. The temperature variation of the initial permeability of these samples was carried out from $30^{\circ} \mathrm{C}$ to $250^{\circ} \mathrm{C}$. The NiCuZn ferrite had higher initial permeability than that of the NiZn ferrite, which could be attributed to the microstructure. Saturation magnetization increases from $40 \mathrm{emug} / \mathrm{g}$ (NiZn) to 47 emug/g (NiCuZn). The dielectric constant $\left(\varepsilon^{\prime}\right)$ and dielectric loss tangent $(\tan \delta)$ of NiZn and NiCuZn ferrite samples decreases with increase in frequency exhibiting normal ferrimagnetic behavior. The NiCuZn ferrite had better electromagnetic properties than the NiZn ferrite.
\end{abstract}

Keywords: Microwave Sintering; Soft Ferrites; Magnetic Properties; Dielectric Properties

\section{Introduction}

The use of microwave radiation in the processing of various ceramic materials such as dielectric, magnetic, superconducting, polymer and other composite materials offers several advantages over conventional processing alternatives. The unique and potential benefits that microwave energy can provide over conventional methods such as rapid, internal and selective heating have stimulated much interest in many researchers to apply this technique in ceramic processing such as firing, annealing and sintering. The direct interaction or coupling between microwaves and the material is responsible for the rapid and internal heating, which makes the energy conversion rather than energy transfer, and as a consequence volumetric heating occurs. The volumetric heating is one of the possible reasons for improvement in microstructure and hence the dielectric and magnetic properties of the materials [1,2].

The poly crystalline ferrites have very important structural, magnetic and electrical properties that are dependent on several factors such as method of preparation, sub-

"Corresponding author. stitution of cations, sintering temperature and time, chemical composition and microstructure, etc. The spinel ferrites with low loss at higher frequencies are used in pulse transformers, inductances, reflection coils, antennas and modulators, etc. The NiZn and MgZn ferrites are suitable for these applications [3]. The Ni-Zn ferrites are considered as the most versatile ferrites for their high resistivity and low eddy current losses. The high frequency applications and further miniaturization of magnetic components enable the use of NiZn or NiCuZn ferrites, because both of them have high electrical resistivity and can miniaturize magnetic components without a bobbin [4-6]. The magnetic properties of these ferrite materials are mainly determined by their chemical composition. The substitution of $\mathrm{CuO}$ to the NiZn ferrite could also lower its sintering temperature and modifies structural and electromagnetic properties $[7,8]$. However, to the best of our knowledge, the detailed comparison of low temperature sintered NiZn and $\mathrm{NiCuZn}$ ferrites has not yet been reported. Only limited researchers have studied the comparison of NiZn and $\mathrm{NiCuZn}$ ferrites with excellent magnetic and electrical properties [8-10]. Therefore, in this paper, we fixed the 
preparing process and the low-temperature sintered NiZn and NiCuZn ferrites, and compared their sintering density, microstructures, magnetic and electric properties.

\section{Experimental Details}

\subsection{Materials}

Starting materials for the synthesis of $\mathrm{NiZn}$ and $\mathrm{NiCuZn}$ ferrites were Ferric Oxide, Nickel Oxide, Zinc Oxide and Copper oxide powders. All the chemicals are purchased from Merck and used as received. All the chemicals and reagents were used without further purification.

\subsection{Preparation of Soft Ferrites}

In this study, a modified microwave oven (Sharp, $1.1 \mathrm{~kW}$, $2.45 \mathrm{GHz}$ ) was used to sinter $\mathrm{NiZn}$ and $\mathrm{NiCuZn}$ ferrite samples. Figure 1 shows the experimental settings in the microwave oven. The general configuration of the microwave furnace has been described elsewhere [11]. The NiZn and $\mathrm{NiCuZn}$ ferrites with the respective compositions of $\mathrm{Ni}_{0.40} \mathrm{Zn}_{0.60} \mathrm{Fe}_{2} \mathrm{O}_{4}$ and $\mathrm{Ni}_{0.35} \mathrm{Cu}_{0.05} \mathrm{Zn}_{0.60} \mathrm{Fe}_{2} \mathrm{O}_{4}$ were prepared using the microwave sintering method. The analytical grade $\mathrm{Fe}_{2} \mathrm{O}_{3}, \mathrm{NiO}, \mathrm{ZnO}$, and $\mathrm{CuO}$ powders were weighed according to the corresponding composition, intimately mixed and the resulting powders were ball milled using a planetary ball mill (Restch PM 200, Germany) in agate bowls with agate balls in acetone medium for $20 \mathrm{~h}$. The slurry was dried and loosely pressed into cakes using a hydraulic press. These cakes were pre-sintered at a temperature of $800^{\circ} \mathrm{C}$ for $4 \mathrm{~h}$ in closed alumina crucibles. The presintered cakes removed from the furnace were crushed and ball milled in an acetone medium in agate bowls with agate balls for another $30 \mathrm{~h}$ to obtain fine particle size. These slurries after drying were sieved to obtain a uniform particle size. The green powder thus obtained was then pressed using a suitable die in the form of toroids of dimensions $1.2 \mathrm{~cm} \mathrm{OD,} 0.8 \mathrm{~cm}$ ID and $0.4 \mathrm{~cm}$ thickness and pellets of dimensions $1.2 \mathrm{~cm}$ diameter and $0.3 \mathrm{~cm}$ thickness with a hydraulic press at a pressure of $200 \mathrm{MPa}$ using 3\% PVA

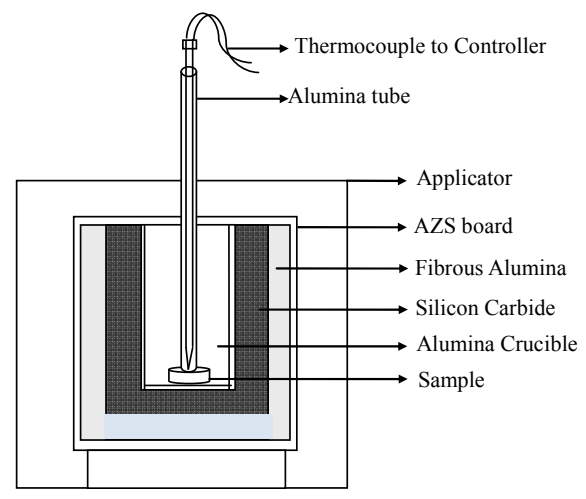

Figure 1. Schematic view of sample environment in the microwave cavity. solution as a binder and the samples were finally sintered at $950^{\circ} \mathrm{C}$ for $30 \mathrm{~min}$ in air at atmospheric pressure.

\subsection{Characterization}

The bulk density was determined using the Archimedes method using distilled water as the immersion fluid. XRD patterns of samples were obtained using an X-ray diffraction system (PM 1730, Germany) using $\mathrm{CuK}_{\alpha}$ radiation. The specimens were polished with $0.3 \mu \mathrm{m}$ alumina powder and thermally etched prior to examination with the help of a scanning electron microscope (SEM, CRL-ZESISEVO-MAI5, Japan). The DC resistivity of these ferrite pellets were evaluated using the Keithley electrometer (Model 6514 , India). The initial permeability, $\left(\mu_{i}\right)$ of these ferrite toroids were evaluated using the standard formulae from the inductance measurements carried out at $10 \mathrm{kHz}$ using a computer controlled impedance analyzer (Hioki Model 3532-50 LCR HiTester, Japan). The magnetic characteristics were measured with VSM-Lakeshore 665 Vibrating sample magnetometer (VSM). These measurements were carried out in the temperature range $30^{\circ} \mathrm{C}-250^{\circ} \mathrm{C}$ at $10^{\circ}$ temperature intervals. The dielectric constant of the samples was calculated from the capacitance $\left(\mathrm{C}_{\mathrm{p}}\right)$ and loss factor $(\tan \delta)$ values measured using impedance analyzer in the frequency range $100 \mathrm{~Hz}$ to $1 \mathrm{MHz}$ at room temperature.

\section{Results and Discussions}

Figure 2 shows the XRD patterns of the NiZn and Ni$\mathrm{CuZn}$ ferrite samples. As we can see from the figure, both the samples possesses single phase with spinel structure. Figure 3 presents the micrographs of the NiZn and Ni$\mathrm{CuZn}$ ferrite samples sintered at $950^{\circ} \mathrm{C}$. The average grain size is observed to be 3.8 and $4.6 \mu \mathrm{m}$ for $\mathrm{NiZn}, \mathrm{NiCuZn}$ ferrites respectively.

The Lattice constant, X-ray density, bulk density, percentage of porosity, average grain size, Curie transition

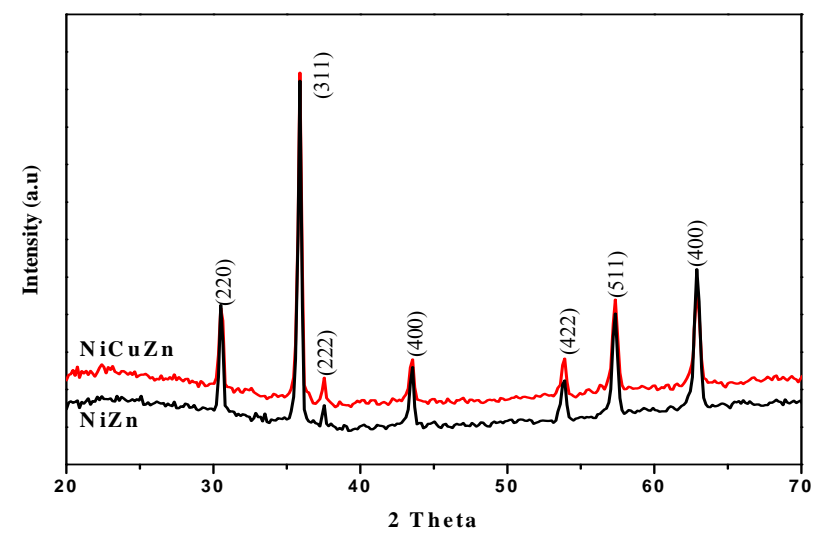

Figure 2. XRD diffraction of NiZn and NiCuZn ferrites sintered at $950^{\circ} \mathrm{C}$. 
temperature and DC resistivity of sintered NiZn and NiCuZn ferrite samples are also tabulated in Table 1. It is seen from this table that the lattice parameter increases from $8.342 \AA$ (NiZn) to $8.392 \AA$ (NiCuZn) [9]. This increase is attributable to the higher ionic of $\mathrm{Cu}^{2+}(0.73 \AA)$ compared to that of $\mathrm{Ni}^{2+}(0.69 \AA)$. As copper content increases at the expense of $\mathrm{Ni}$, the lattice seems to expand slightly to accommodate the increased number of $\mathrm{Cu}^{2+}$ ions of relatively larger radius resulting in increased value of lattice parameter. It can also be observed from the Table 1, that X-ray density of each sample (NiZn and NiCuZn ferrite) is higher than the corresponding bulk density of sintered smples. The variation of room-temperature resistivity with $\mathrm{Cu}$ content (Table 1) shows that resistivity decreases with addition of $\mathrm{Cu}$ content to NiZn ferrite.

The magnetic initial permeability $\left(\mu_{i}\right)$ as a function of temperature (at constant frequency, $10 \mathrm{kHz}$ ) from room temperature to Curie transition temperature was also studied. The temperature variation of magnetic initial permeability for both samples is shown in Figure 4. It is evident from figure that the magnetic initial permeability remains constant over a wide range for the microwave sintered sample. It can also be noticed from the figure that the microwave sintered ferrites show good thermal stability. This indicates that the shape of the permeability-temperature curves depends on the preparation conditions. It can be noted from Figure 4 that as the temperature increases the magnetic initial permeability remains constant up to a certain temperature and increases to a peak value and then abruptly falls to a minimum value. The temperature at which this abrupt fall takes place is the magnetic Curie transition temperature $\left(T_{c}\right)$. At this temperature the specimens transform from the ferrimagnetic phase to the paramagnetic phase. An increase in NiCuZn ferrite initial permeability is observed compared with NiZn-ferrite. The magnetic initial permeability for the material is expected to strongly depend on the microstructure, as the magnetic initial permeability represents the mobility of magnetic domain wall in response to the small applied field [12]. It is also seen from Figure 2 that the NiCuZn samples result in large-grain samples leading to enhancement in permeability.

Figure 5 shows that $\mathrm{Cu}$ addition increases saturation magnetization, probably by increasing grain size and crystallinity at the same temperature treatment compared to NiZn-ferrite. Saturation magnetization $\left(M_{s}\right)$ values are comparable to those reported by other authors $[13,14]$.
Coercivity is related to the variation of the particle size. The substitution of nickel by copper also affects bulk density and resistivity of the ferrite (Table 1). The increase in density is attributed to the increase in grain size and reduction of the pores in the ferrite microstructure as seen in SEM microphotography (Figure 3).

The dielectric properties such as dielectric constant, dielectric loss $(\tan \delta)$ are important for multilayer chip inductors used in high frequency range. The frequency dependence of dielectric constant for microwave sintered $\mathrm{NiZn}$ and NiCuZn ferrite samples is shown in Figures 6(a) and (b). The plots illustrate that the dielectric constant decreases with increasing frequency reaching constant value

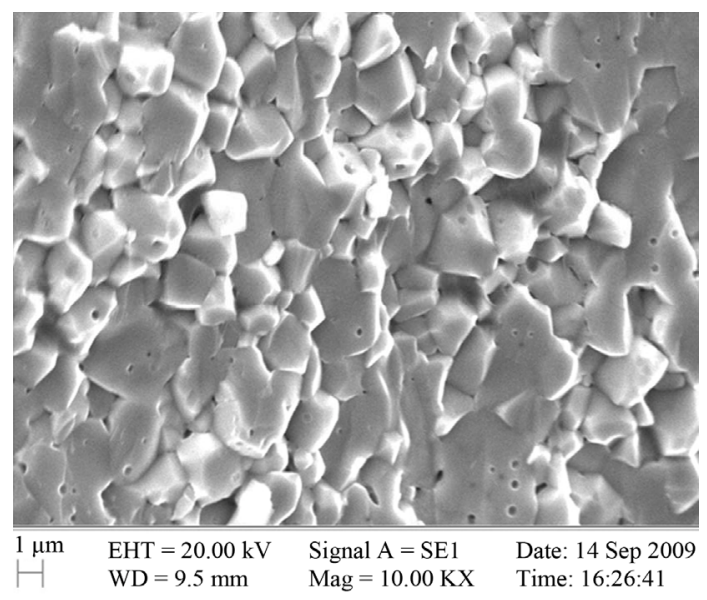

(a)

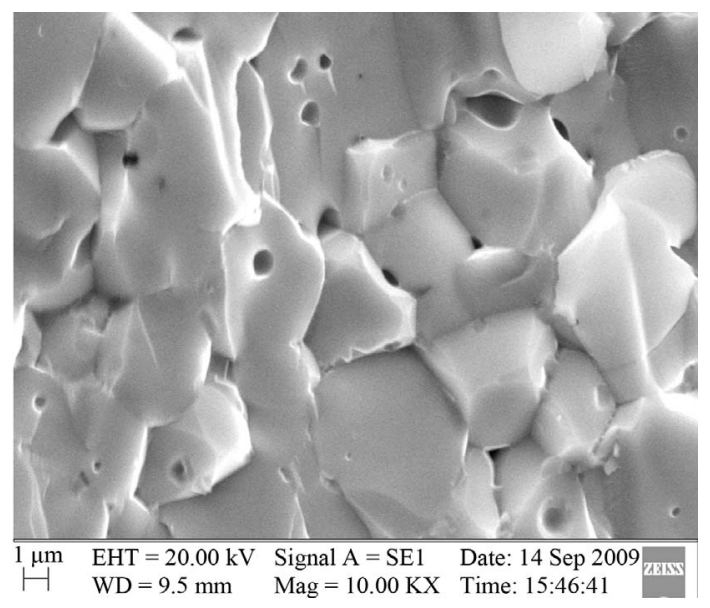

(b)

Figure 3. SEM images of the NiZn and NiCuZn ferrites sintered at $950^{\circ} \mathrm{C}$. (a) NiZn ferrite; (b) NiCuZn ferrite.

Table 1. Lattice constant, $X$-ray density, bulk density, porosity, average grain size, Curie transition temperature and DC resistivity for the $\mathrm{NiZn}$ and $\mathrm{NiCuZn}$ ferrites sintered at $950^{\circ} \mathrm{C}$.

\begin{tabular}{cccccccc}
\hline Ferrites & $\mathrm{a}(\AA)$ & $\mathrm{d}_{\mathrm{x}}\left(\mathrm{gm} / \mathrm{cm}^{3}\right)$ & $\mathrm{d}_{\mathrm{B}}\left(\mathrm{gm} / \mathrm{cm}^{3}\right)$ & $\mathrm{P}(\%)$ & $\mathrm{G}_{\mathrm{avg}}(\mu \mathrm{m})$ & $\mathrm{T}_{\mathrm{c}}\left({ }^{\circ} \mathrm{C}\right)$ & $\rho(\Omega \mathrm{cm})$ \\
\hline $\mathrm{NiZn}$ & 8.342 & 5.342 & 4.927 & 7.8 & 3.8 & 160 & $5.2 \times 10^{8}$ \\
$\mathrm{NiCuZn}$ & 8.392 & 5.365 & 5.034 & 6.1 & 4.6 & 150 & $3.7 \times 10^{8}$ \\
\hline
\end{tabular}




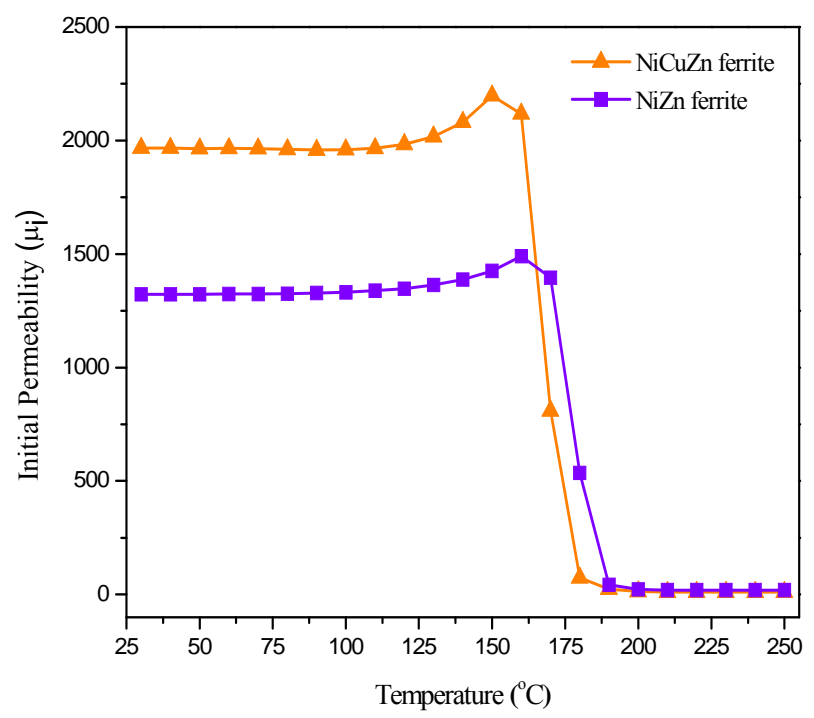

Figure 4. Temperature variation of initial permeability of NiZn and NiCuZn ferrite samples.

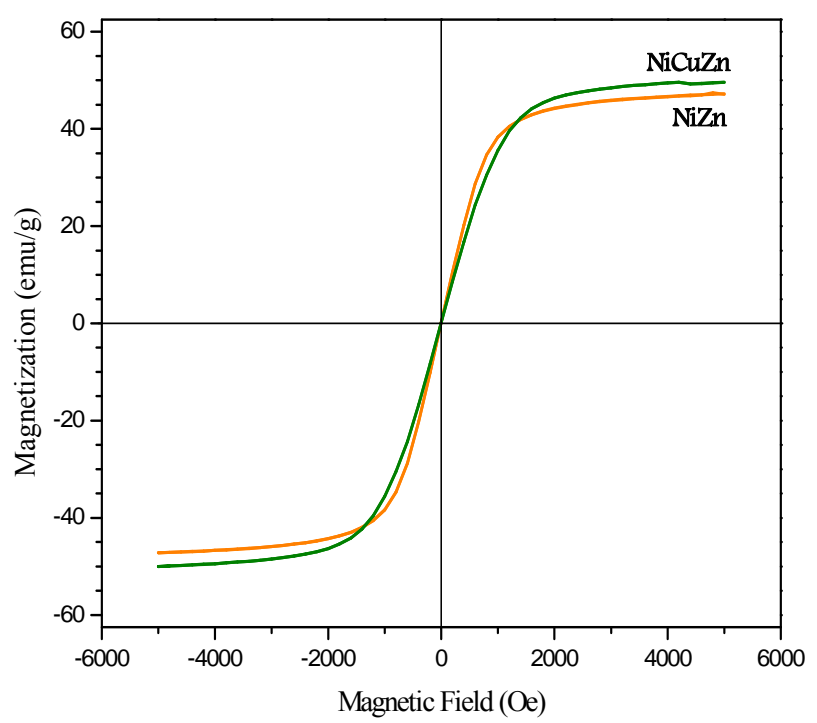

Figure 5. Room temperature M-H graphs of NiZn and NiCuZn ferrite samples.

at higher frequencies. The variation reveals the dispersion due to Maxwell-Wanger [15,16] type interfacial polarization in agreement with Koop's phenomenological theory [17]. This is the normal behavior for ferrites [18]. This is similar to the results reported earlier for $\mathrm{NiZn}$ [19], Ni-Cu [20] and Ni-Cu-Zn [9] ferrites. An important decrease of dielectric loss $(\tan \delta)$ with frequency is observed. The low loss values $(0.03)$ at higher frequencies show the potential of these samples for high-frequency applications. As seen in the figure, the dielectric constant for $\mathrm{NiCuZn-ferrite} \mathrm{is} \mathrm{higher} \mathrm{than} \mathrm{for} \mathrm{NiZn} \mathrm{ferrite.} \mathrm{The}$ mechanism of polarization in polycrystalline ferrites is mainly reported to be hopping of electrons between ions of the same element but in different oxidation states [21].

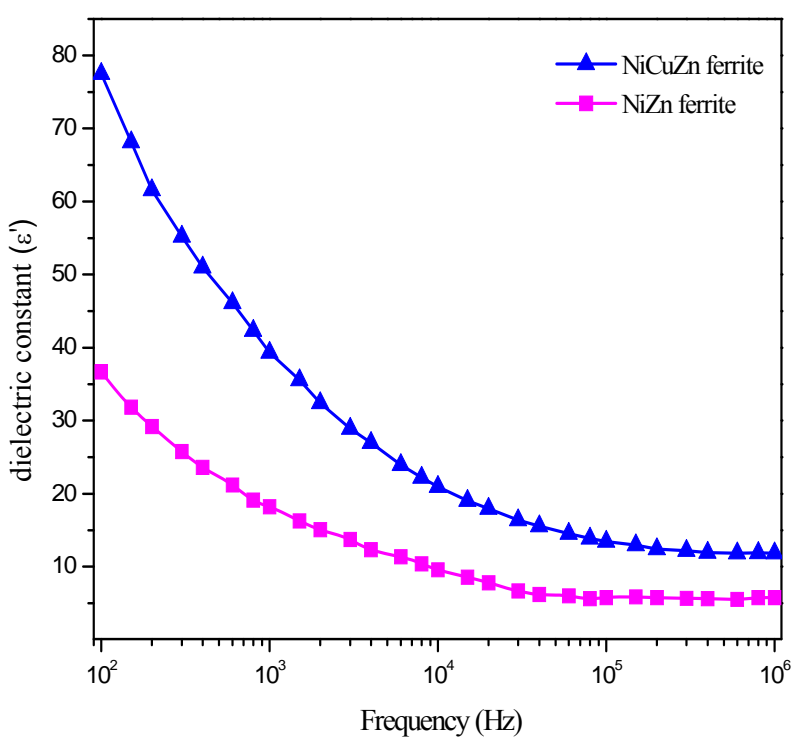

(a)

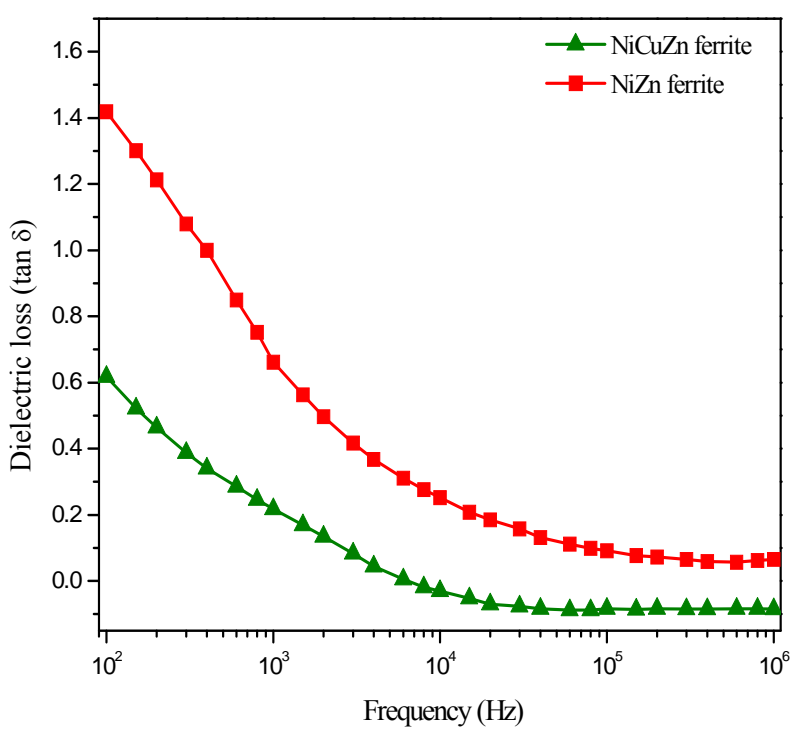

(b)

Figure 6. (a) Dielectric constant $\left(\varepsilon^{\prime}\right)$ as a function of frequency of NiZn and NiCuZn ferrite samples; (b) Dielectric loss $(\tan \delta)$ as a function of frequency of NiZn and NiCuZn ferrite samples.

\section{Conclusions}

In this paper, we have compared the lattice parameter, sintering density, microstructures, magnetic and dielectric properties of the low temperature sintered NiZn and Ni$\mathrm{CuZn}$ ferrites, and the following conclusions can be drawn when the substitution of $\mathrm{Cu}$ in NiZn ferrite.

1) The lattice parameter increases with the copper content due to larger ionic radius of $\mathrm{Cu}^{2+}$ compared to $\mathrm{Ni}^{2+}$;

2) A remarkable increase in the value of bulk density has been found with $\mathrm{Cu}$ substitution for $\mathrm{Ni}$ in $\mathrm{NiZn}$ ferrite;

3) The average grain size is found to be high with the 
substitution of $\mathrm{Cu}$ in NiZn ferrite;

4) $\mathrm{Cu}$ substitution in NiZn-ferrite enhances initial permeability and saturation magnetization. This is mainly attributed to the presence of $\mathrm{Cu}$ ions activating the sintering processes in ferrites and leading to increase in density;

5) The dielectric constant and loss tangent are found to decrease with increase in frequency. Dielectric loss $(\tan \delta)$ is of the order $10^{-2}$.

\section{REFERENCES}

[1] P. K. Patro, A. R. Kulkarni, S. M. Gupta and C. S. Harendranath, "Improved Microstructure, Dielectric and Ferroelectric Properties of Microwave-Sintered

$\mathrm{Sr}_{0.5} \mathrm{Ba}_{0.5} \mathrm{Nb}_{2} \mathrm{O}_{6}$," Physica B: Condensed Matter, Vol. 400, No. 1-2, 2007, pp. 237-242. doi:10.1016/j.physb.2007.07.022

[2] Z. Xie, J. Yang, X. Huang and Y. Huang, "Microwave Processing and Properties of Ceramics with Different Loss," Journal of European Ceramic Society, Vol. 19, No. 3, 1999, pp. 381-387. doi:10.1016/S0955-2219(98)00203-9

[3] V. R. K. Murty and B. Vishwanathan, "Ferrites Materials: Science and Technology," Narosa Publishing House, Mumbai, 1990.

[4] Y. Matsuo, M. Inagaki, T. Tomozawa and F. Nakao, "High Performance NiZn Ferrite," IEEE Transactions on Magnetics, Vol. 37, No. 4, 2001, pp. 2359-2361. doi:10.1109/20.951172

[5] K. Kondo, T. Chiba, S. Yamada and E. Otsuki, "Analysis of Power Loss in Ni-Zn Ferrites," Journal of Applied Physics, Vol. 87, No. 9, 2000, pp. 6229-6231. doi: $10.1063 / 1.372663$

[6] A. C. F. M. Costa, E. Tortella, M. R. Morelli and R. H. G. A. Kiminami, "Synthesis, Microstructure and Magnetic Properties of Ni-Zn Ferrites," Journal of Magnetism and Magnetic Materials, Vol. 256, No. 1-3, 2003, pp. 174-182. doi:10.1016/S0304-8853(02)00449-3

[7] S. R. Murthy, "Low Temperature Sintering of NiCuZn Ferrite and Its Electrical, Magnetic and Elastic Properties," Journal of Materials Science Letters, Vol. 21, No. 8, 2002, pp. 657-660. doi:10.1023/A:1015608625798

[8] P. A. Jadhav, R. S. Devan, Y. D. Kolekar and B. K. Chougule, "Structural, Electrical and Magnetic Characterizations of Ni-Cu-Zn Ferrite Synthesized by Citrate Precursor Method," Journal of Physics and Chemistry of Solids, Vol. 70, No. 2, 2009, pp. 396-400. doi:10.1016/j.jpcs.2008.11.019

[9] J. C. Aphesteguy, A. Damiani, D. D. Giovanni and S. E. Jacobo, "Microwave-Absorbing Characteristics of Epoxy Resin Composites Containing Nanoparticles of NiZn- and NiCuZn-Ferrites," Physica B: Condensed Matter, Vol. 404, No. 18, 2009, pp. 2713-2716.

doi:10.1016/j.physb.2009.06.065
[10] H. Su, H. Zhang, X. Tang, Y. Jing and Y. Liu, "Effects of Composition and Sintering Temperature on Properties of NiZn and NiCuZn Ferrites," Journal of Magnetic Materials, Vol. 310, No. 1, 2007, pp. 17-21. doi:10.1016/j.jmmm.2006.07.022

[11] M. Penchal Reddy, W. Madhuri, N. Ramamanohar Reddy, K. V. Siva Kumar, V. R. K. Murthy and R. Ramakrishna Reddy, "Magnetic Properties of Ni-Zn Ferrites Prepared by Microwave Sintering Method," Journal of Electroceramics, Vol. 28, No. 28, 2012, pp. 1-9.

[12] C. Y. Tsay, K. S. Liu and I. N. Lin, "Microwave Sintering of $\left(\mathrm{Bi}_{0.75} \mathrm{Ca}_{1.2} \mathrm{Y}_{1.05}\right)\left(\mathrm{V}_{0.6} \mathrm{Fe}_{4.4}\right) \mathrm{O}_{12}$ Microwave Magnetic Materials," Journal of the European Ceramic Society, Vol. 24, No. 6, 2004, pp. 1057-1061. doi:10.1016/S0955-2219(03)00401-1

[13] M. C. Dimri, A. Verma, S. Kashyap, D. C. Dube, O. P. Thakur and Ch. Prakash, "Structural, Dielectric and Magnetic Properties of NiCuZn Ferrite Grown by Citrate Precursor Method," Materials Science and Engineering B, Vol. 133, No. 1-3, 2006, pp. 42-48. doi:10.1016/j.mseb.2006.04.043

[14] E. Rezlescu, L. Sachelarie, P. D. Popa and N. Rezlescu, "Effect of Substitution of Divalent Ions on the Electrical and Magnetic Properties of Ni-Zn-Me Ferrites," IEEE Transactions on Magnetics, Vol. 36, No. 6, 2000, pp. 2841-2846. doi:10.1109/20.914348

[15] K. C. Maxwell, "Electricity and Magnetism," Oxford University Press, London, Vol. 33, 1873, p. 328.

[16] K. W. Wagner, "Zur Theorie der Unvolkommenen Dielektrika," American Physics, Vol. 40, 1913, p. 817.

[17] C. G. Koops, "On the Dispersion of Resistivity and Dielectric Constant of Some Semiconductors at Audio Frequencies," Physical Review, Vol. 83, 1953, pp. 121-125. doi:10.1103/PhysRev.83.121

[18] K. Katsmi, S. Mamoru, I. Tatrsuo and I. Katsuya, "Dielectric Behavior of Water Molecules Adsorbed on Iron(III) Oxide Hydroxides," Bulletin of the Chemical Society of Japan, Vol. 48, No. 6, 1975, pp. 1764-1767. doi:10.1246/bcsj.48.1764

[19] P. Yadoji, R. Peelamedu, D. Agarwal and R. Roy, "Microwave Sintering of Ni-Zn Ferrites: Comparison with Conventional Sintering," Materials Science and Engineering B, Vol. 98, No. 3, 2003, pp. 269-278. doi:10.1016/S0921-5107(03)00063-1

[20] D. R. Patil and B. K. Chougule, "Effect of Copper Substitution on Electrical and Magnetic Properties of $\mathrm{NiFe}_{2} \mathrm{O}_{4}$ ferrite," Materials Chemistry and Physics, Vol. 117, No. 1, 2009, pp. 35-40. doi:10.1016/j.matchemphys.2008.12.034

[21] N. Rezlescue and E. Rezlescue, "Dielectric Properties of Copper Containing Ferrites," Physica Status Solid (a), Vol. 23, No. 2, 1974, pp. 575-582. doi:10.1002/pssa.2210230229 